\title{
MAGNETIC FIELDS IN CORE COLLAPSE SUPERNOVAE: POSSIBILITIES AND GAPS
}

\author{
J. CRAIG WHEELER AND SHIZUKA AKIYAMA \\ Department of Astronomy University of Texas \\ E-mail: wheel@astro.as.utexas.edu; shizuka@astro.as.utexas.edu
}

\begin{abstract}
Spectropolarimetry of core collapse supernovae has shown that they are asymmetric and often, but not universally, bi-polar. The Type IIb SN 1993J and similar events showed large scatter in the Stokes parameter plane. SN 2002ap which showed very high photospheric velocities in early phases revealed that the dominant axes associated with hydrogen, with oxygen, and with calcium were all oriented substantially differently. Observational programs clearly have much more to teach us about the complexity of asymmetric supernovae and the physics involved in the asymmetry. Jet-induced supernova models give a typical jet/torus structure that is reminiscent of some objects like the Crab nebula, SN 1987A and perhaps Cas A. Jets, in turn, may arise from the intrinsic rotation and magnetic fields that are expected to accompany core collapse. We summarize the potential importance of the magneto-rotational instability for the core collapse problem and sketch some of the effects that large magnetic fields, $\sim 10^{15} \mathrm{G}$, may have on the physics of the supernova explosion. Open issues in the problem of multi-dimensional magnetic core collapse are summarized and a critique is given of some recent MHD collapse calculations. A crucial piece of information that can inform the discussion of potential MHD effects even in the absence of the explicit inclusion of magnetic fields is to give sufficient information from a rotating collapse to at least crudely estimate the time-dependent saturation field according to the prescription $v_{a} \sim r \Omega$. Many studies of rotating collapse produce such information, but fail to present it explicitly.
\end{abstract}

\section{Introduction}

Spectropolarimetry of supernovae has opened up a new window on these spectacular events and yielded remarkable new insights. A few rare, nearby supernovae and supernova remnants have revealed asymmetric images. Among these are the Crab nebula with its prominent jet/torus structure revealed by CXO, SN 1987A (Wang et al. 2002a) and Cas A (Fesen 2001; Hwang et al. 2004). It was not clear on this limited basis whether or not the strong asymmetries of these objects was important to the intrinsic process of the explosion. Spectropolarimetry has extended our knowledge of 
the composition-dependent geometry of core-collapse supernovae to numerous extragalactic supernovae. Spectropolarimetry of supernovae probes the geometrical structure of matter shed by a star before it explodes and the structure of the ejecta of the explosion with an effective spatial resolution far superior to any envisaged optical interferometry (Wang et al. 2002b). The structure revealed is closely related to the explosion mechanisms and to the progenitor systems.

Spectropolarimetry of supernovae continues to show that all corecollapse supernovae (those associated with young populations; Type II, Type $\mathrm{Ib} / \mathrm{c}$ ) are polarized and hence substantially asymmetric (Wang et al. 1996; Wang et al. 2001, 2002a,b, 2003a,b; Leonard et al. 2000; Leonard \& Filippenko 2001; Leonard et al. 2001, 2002). The understanding that corecollapse supernovae are routinely asymmetric developed in parallel with the discovery that gamma-ray bursts are highly-collimated events. This supernova/gamma-ray burst connection was dramatically confirmed when SN 2003dh was revealed in the afterglow of GRB 030329 (Stanek et al. 2003; Hjorth et al. 2003; Kawabata et al. 2003).

Here we summarize some of the background on spectropolarimetry of core-collapse supernovae and the evidence that they are generically asymmetric. We discuss the importance of the magneto-rotational instability for the collapse problem and some of the attendant physics that may be expected. We outline some of the important issues involved in doing MHD collapse and give a summary and critique of some recent attempts to merge MHD physics with core collapse physics.

\section{Results of Spectropolarimetry}

The first qualitative insight of the "Texas" program of routine spectropolarimetry was that there is a distinct difference between Type Ia supernovae and core collapse events: Type II, Type IIn, Type IIb, Type Ib and Type Ic. The first systematic study (Wang et al. 1996) showed that core collapse supernovae are substantially polarized at the $1 \%$ level, but that Type Ia were generally substantially less polarized. As more data were added, it became clear that the polarization of the core-collapse supernovae was deeply intrinsic to the explosion mechanism. The polarization grows as the photosphere recedes into the ejecta and tends to be higher for events with less thick blanketing hydrogen envelopes (Wang et al. 1996, 2001; Leonard et al. 2001), This implies that the basic machine that powers the explosion is asymmetric. Polarization of $\sim 1 \%$ implies an axis ratio of about 2 to 1 
if interpreted in terms of ellipsoids of rotation (Höflich 1991).

The data often show a well-defined orientation suggesting that the explosion was substantially bi-polar. Figure 1 shows the data for the Type II plateau event SN 1999em. The data fall on the same line in the Stokes parameter plane as a function of time and of wavelength. This shows that there is a strongly favored axis to the geometry, hence that it is substantially bi-polar, a pattern repeated in several other events. We stress that there are exceptions. The Type IIb SN 1993J and the very similar event SN 1996cb showd large scatter in the Stokes parameter plane (Wang et al. 2001). SN 2002ap, a Type Ic that showed very high photospheric velocities in early phases revealed that the dominant axes associated with hydrogen, with oxygen, and with calcium were all oriented substantially differently (Wang et al. 2003b). Observational programs clearly have much more to teach us about the complexity of asymmetric supernovae and the physics involved in the asymmetry.

\section{Asymmetric Core Collapse}

We have learned that all core collapse supernovae are substantially asymmetric and often bi-polar. This alone does not prove that supernovae are exploded by jets, but numerical simulations (Khokhlov et al. 1999; Khokhlov \& Höflich 2001; Höflich, Wang \& Khokhlov 2001) have shown that bi-polar jets can, in principle, explode supernovae and produce these asymmetries with no aid from the classical powering process of neutrino deposition. The origin of any such jets remains a mystery. Rotation alone can induce asymmetric neutrino fluxes (Shimizu, Yamada, \& Sato 1994; Fryer \& Heger 2000 ), but rotation will inevitably lead to magnetic field amplification that can both produce MHD effects, including possibly jets (Wheeler et al. 2000, 2002; Akiyama et al. 2003), and affect neutrino transport (see $\S 9$ for a brief discussion). In practice, neutrino transport, probably itself asymmetric and bi-polar will remain an important ingredient in the phenomenon.

Asymmetries will also affect nucleosynthesis (Maeda et al. 2002; Nagataki et al. 2003). An important aspect of the jet-induced simulations is a characteristic feature of the chemical distribution. There will be a generic tendency for the iron-peak elements to be ejected along the jet direction with the traditional elements of bulk nucleosynthesis (oxygen, calcium) being ejected predominantly in the equatorial plane. There is evidence that SN 1987A shows just that sort of configuration (Wang et al. 2002a). An interesting challenge to this picture is the recent data from a long CXO 


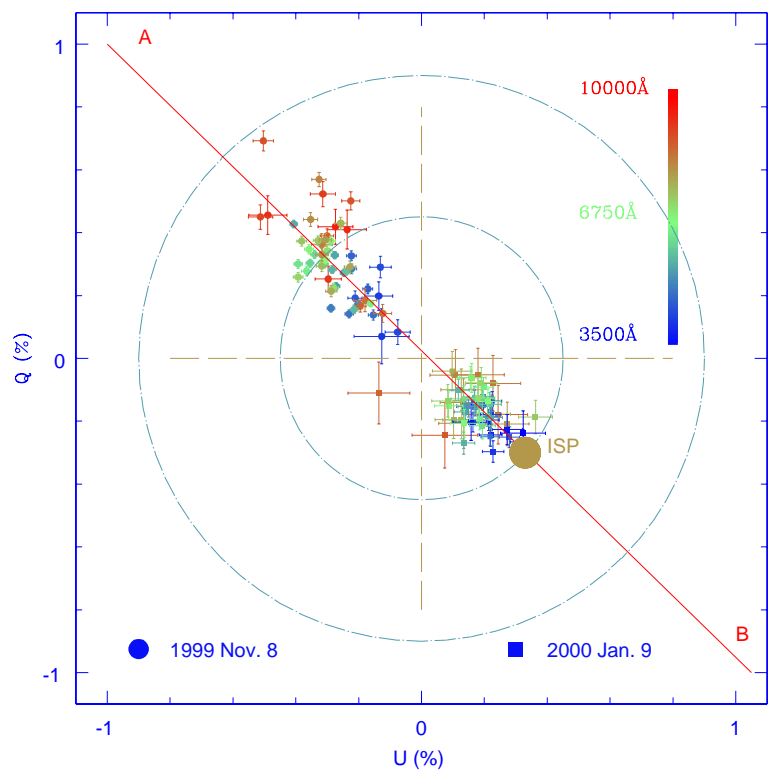

Figure 1. Two epochs of spectropolarimetry on the Type II plateau supernovae 1999em showing the bi-polar nature of the ejecta that falls along a single locus in the plane of the Stokes vectors as a function of time and wavelength (Wang et al. 2001)

exposure of Cas A that clearly shows the jet and counter-jet structure long associated with Cas A, but predominantly in the element silicon, not, apparently, iron (Hwang et al. 2004). Another challenge is the displacement of the central compact object to the south of the center of expansion of the remnant, implying a "kick" to the compact object of about $330 \mathrm{~km} \mathrm{~s}^{-1}$ roughly normal to the locus of the jet (Thorstensen, Fesen \& van den Bergh, 2001). It is possible that this complex dynamical structure is related to the multiple axes revealed in SN 2002ap (Wang et al. 2003b).

The tendency for collapse explosions to be bi-polar suggests that at the very least rotation is involved to provide a special, well-defined axis. There are strong arguments that rotation will naturally and unavoidably be attended with dynamo processes that generate and amplify magnetic fields. It is probably inconsistent to consider rotation in either the collapse process or the stellar evolution that precedes it without simultaneously and self-consistently considering the attendant magnetic field. 
The ultimate problem of core collapse is one of three dimensions, rotation, magnetic fields, and neutrino transport. We have suspected this all along, but the polarization of supernovae and jets from GRBs demands that the issue of substantial asymmetries be met head on.

\section{The Magneto-Rotational Instability and Core Collapse}

An important physical effect that must be considered in the context of core collapse is the magneto-rotational instability (MRI; Balbus \& Hawley 1991, 1998). Core collapse will lead to strong differential rotation near the surface of the proto-neutron star even for initial solid-body rotation of the iron core (Kotake, Yamada \& Sato 2003; Ott et al. 2003). The criterion for instability to the MRI is a negative gradient in angular velocity, as opposed to a negative gradient in angular momentum for the Rayleigh dynamical instability. This condition is generally satisfied at the surface of a newly formed neutron star during core collapse and so the growth of magnetic field by the action of the MRI is inevitable.

More quantitatively, when the magnetic field is small and/or the wavelength is long $\left(\mathrm{k} \mathrm{v}_{\mathrm{a}}<\Omega\right)$ the instability condition can be written (Balbus \& Hawley 1991, 1998):

$$
N^{2}+\frac{\partial \Omega^{2}}{\partial \ln \mathrm{r}}<0
$$

where $\mathrm{N}$ is the Brunt-Väisälä frequency. Convective stability will tend to stabilize the MRI, and convective instability to reinforce the MRI. The saturation field given by general considerations and simulations is approximately given by the condition: $\mathrm{v}_{\mathrm{a}} \sim \lambda \Omega$ where $\lambda \lesssim \mathrm{r}$ or $\mathrm{B}^{2} \lesssim 4 \pi \rho \mathrm{r}^{2} \Omega^{2}$ where $\mathrm{v}_{\mathrm{a}}$ is the Alfvén velocity.

Akiyama et al. (2003) have presented a proof-of-principle calculation that the physics of the MRI is inevitable in the context of the differentiallyrotating environment of proto-neutron stars. The great power of the MRI to generate magnetic field is that while it works on the rotation time scale of $\Omega^{-1}$ (as does simple field-line wrapping), the strength of the field grows exponentially. This means that from a plausible seed field of $10^{10}$ to $10^{12}$ $\mathrm{G}$ that might result from field compression during collapse, only $\sim 7$ - $12 \mathrm{e}-$ folds are necessary to grow to a field of $10^{15} \mathrm{G}$. Akiyama et al. (2003) have shown that for rotation that is at all times sub-Keplerian, this instability will naturally grow any seed field exponentially rapidly to a saturation level of order $10^{15}$ to $10^{16} \mathrm{G}$ in a few 10 s of milliseconds, a timescale longer than 
the initial bounce timescale, but much less than popular late-time neutrinoheating mechanisms that work over hundreds of milliseconds.

Figure 2 shows the expected evolution of the angular velocity profile, the magnetic field and the associated MHD luminosity. The portion of the structure with decreasing angular velocity with radius, a generic feature at the boundary of the rotating proto-neutron star, represents structure that is unstable to the magneto-rotational instability. The predicted magnetic field is much larger than the quantum electrodynamic limit of $\sim 10^{13} \mathrm{G}$, but still smaller than the fields that would be directly dynamically important, of order $10^{17}$ to $10^{18} \mathrm{G}$. It remains to be seen whether this level of magnetic field will contribute substantially to asymmetries and jet formation in the explosions. The effects on the equation of state are estimated to be negligible near the PNS where the density is high (Duan 2004; Akiyama et al. 2004), but if a highly magnetic bubble is convected to a low density region, there could be important effects. There could also be effects on the neutrino cross sections as outlined briefly in $\S 7$. We note that these calculations have not yet considered the de-leptonization phase when the neutron star contracts and spins even faster, perhaps producing even larger fields on timescales of seconds.

The resulting characteristic MHD luminosity (cf. Blandford \& Payne 1982) is:

$$
\mathrm{L}_{\mathrm{MHD}} \sim \mathrm{B}^{2} \mathrm{r}^{3} \Omega / 2 \sim 3 \times 10^{52} \mathrm{erg} \mathrm{s}^{-1} \mathrm{~B}_{16}^{2} \mathrm{R}_{\mathrm{NS} .6}^{3}\left(\frac{\mathrm{P}_{\mathrm{NS}}}{10 \mathrm{~ms}}\right)^{-1}
$$

If this power can last for a significant fraction of a second, a supernova could result. The energy of rotation is approximately

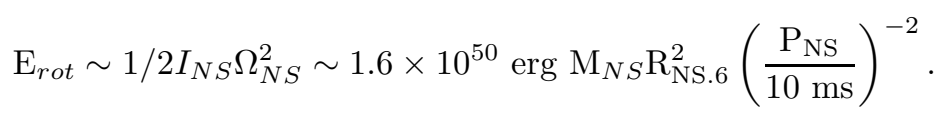

A sufficiently fast rotation of the original iron core is needed to provide ample rotation energy. This will also promote a strong MHD luminosity.

For collapse to form a black hole, the velocities will be Keplerian and the associated, dynamo-driven, predominantly toroidal field will have a saturation strength, $\mathrm{B}^{2} \sim 4 \pi \rho \lambda^{2} \Omega^{2}$ with $\lambda \lesssim \mathrm{r}$, of order $\mathrm{B} \sim 10^{16} \mathrm{G} \rho_{10}^{1 / 2}$ assuming motion, including the Alfvén speed, near the speed of light near the Schwarzschild radius and a characteristic density of order $10^{10} \mathrm{~g} \mathrm{~cm}^{-3}$ (MacFadyen \& Woosley 1999). Fields this large could affect both the dynamics and the microphysics in the black hole-formation problem. Because of the nearly Keplerian motion in the black hole case, the fields generated will be much closer to pressure equipartition than in the neutron star case, 

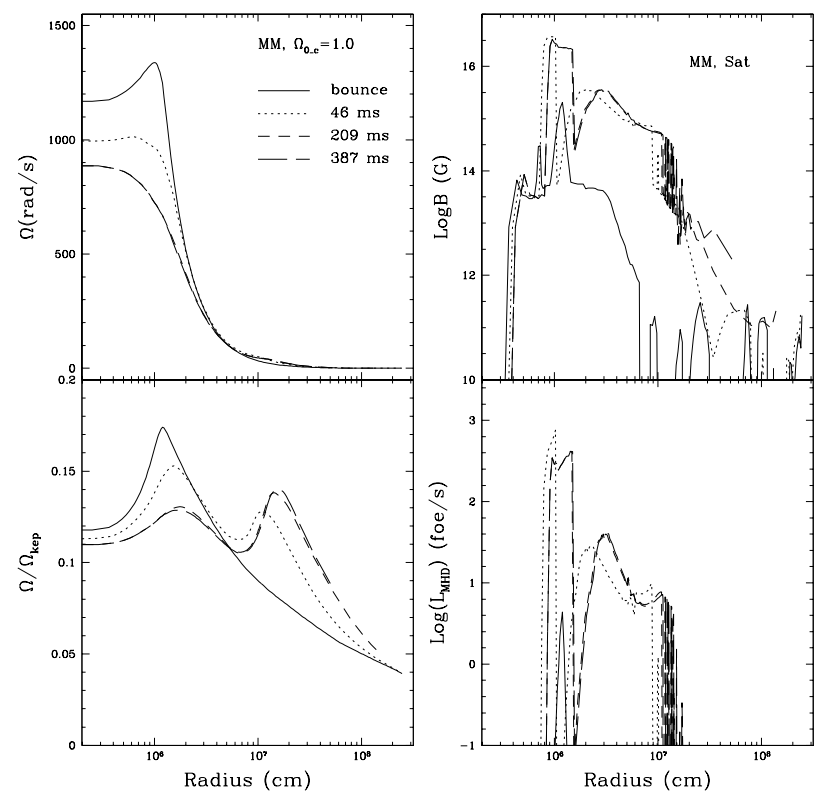

Figure 2. Angular velocity, field strength and MHD luminosity(in units of $10^{51} \mathrm{erg} \mathrm{s}^{-1}$ ) for a representative initial differential rotation of the iron core as a function of time from Akiyama et al. (2003)

and hence, perhaps, even more likely to have a direct dynamical effect. The associated MHD power in the black hole case would be roughly $10^{52}-10^{53}$

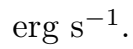

The implication of this work is that the MRI is probably unavoidable in the differentially rotating ambience of core collapse for either "ordinary" supernovae and for those that produce gamma-ray bursts. Calculations that omit this physics are probably incorrect at some level. The magnetic field generated by the MRI should be included in any self-consistent calculation, but issues of how to capture this physics in numerical calculations are challenging. Balbus \& Hawley (1998) summarize their work showing that the specific outcome of MRI calculations depends on the initial field configuration. In $2 \mathrm{D}$, an initial magnetic field aligned with the rotation axis will give a streaming instability, whereas a configuration with a finite RMS field but with zero mean field will give a chaotic, turbulent field. We return to this point below $(\S 8)$. 
These implications need to be explored in much greater depth, but there is at least some possibility that the MRI may lead to strong MHD jets by the magneto-rotational (Meier, Koide \& Uchida 2001) or other mechanisms. A key point is that the relevant dynamics will be dictated by strong, predominantly toroidal fields that are generated internally, and are not necessarily the product of twisting of external field lines that is the basis for so much work on MHD jet and wind mechanisms. Understanding the role of these internal toroidal fields in producing confining coronae (Hawley \& Balbus 2002) or jets (Williams, 2003), in providing the ultimate dipole field strength for both ordinary pulsars and magnetars (Duncan \& Thompson 1992), in setting the "initial" pulsar spin rate after the supernova dissipates (that is, the "final" spin rate from the supernova dynamicists point of view), and any connection to GRBs is in its infancy.

\section{Open Issues}

There are a large number of important open issues. Chief among them are whether or not the rotation and magnetic fields associated with core collapse lead to sufficiently strong MHD jets or other flow patterns to explode supernovae. This issue touches on many others:

- Magnetic effects in the rotating progenitor star

- Dynamos and saturation field strengths

- Effect of large fields on the equation of state

- Effect of large fields on the neutrino cross sections and transport

- Effect of large fields on structure and evolution of the neutron star

- Effect of large fields on jet formation

- Relevance of MRI and field generation to GRBs and "hypernovae"

\section{Dynamo Theory and Saturation Fields}

In traditional mean field dynamo theory, the turbulent velocity field that drives the "alpha" portion of the $\alpha-\Omega$ dynamo was specified and held fixed, but the turbulent velocity field cannot be constant. The buildup of small scale magnetic field tends to inhibit turbulence, cutting off the dynamo process for both small and large scale fields. Since the small scale field tended to grow faster than the large scale field, it appeared that the growth of the large scale field would be suppressed (Kulsrud \& Anderson 1992; Gruzinov \& Diamond 1994). In these theories, the magnetic field energy cascades to smaller length scales where it is ultimately dissipated at the resistive scale. Large scale fields tend to build up slowly, if at all. 
A proposed solution to this problem has been the recognition (Blackman \& Field 2000; Vishniac \& Cho 2001; Field \& Blackman 2002; Blackman \& Brandenburg, 2002; Blackman \& Field 2002; Kleeorin et al. 2002) that the magnetic helicity, $\mathbf{H}=\mathbf{A} \cdot \mathbf{B}$ is conserved in ideal MHD and that this conservation had not been treated explicitly in mean field dynamo theory. Incorporation of this principle leads to an "inverse cascade" of helical field energy to large scales that is simultaneous with the cascade of helical field energy from the driving scale to the dissipation scale. Basically, the large scale helical field and inverse cascade must exist with opposite magnetic helicity to that of the field cascading to small scale. The result (Blackman \& Brandenburg 2002) is the rapid growth of large scale field in a kinematic phase (prior to significant back-reaction) to a strength where the field on both large and small scales is nearly in equipartition with the turbulent energy density. At that point, the back reaction sets in and there tends to be a slower growth to saturation at field strengths that can actually somewhat exceed the turbulent energy density. It may be that the early, fast, kinematic growth is the only phase that is important for astrophysical dynamos, especially in situations that have open boundaries so that field can escape (Brandenburg, Blackman \& Sarson 2003; Blackman \& Tan 2003) and that are very dynamic. The collapse ambience is clearly one of those situations.

A related insight is that the rapid kinematic phase of field growth can lead to magnetic helicity currents (Vishniac \& Cho 2001). It is possible that these magnetic helicity currents can transport power out of the system in twisting, propagating magnetic fields. This is clearly reminiscent of jets or winds, but the physics is rather different than any that has been previously explored in driving jets or winds. This physics needs to be explored in the context of supernovae and gamma-ray bursts.

Vishniac \& Cho (2001) argue that along with conservation of magnetic helicity, $\mathbf{H}=\mathbf{A} \cdot \mathbf{B}$, and the inverse cascade of magnetic field energy to large scales, one will get a current of magnetic helicity that can be crudely represented by

$$
J_{\mathrm{H}} \sim \mathrm{B}^{2} \lambda \mathrm{v}
$$

where the characteristic length, $\lambda$, might be comparable to a pressure scale height, $\ell_{\mathrm{P}}=(\mathrm{d} \ln \mathrm{P} / \mathrm{dr})^{-1}$, and $\mathrm{v} \sim \mathrm{v}_{\mathrm{a}} \sim \ell_{\mathrm{P}} \Omega$. The energy flux associated with this magnetic helicity current is $\mathrm{J}_{\mathrm{H}} / \lambda \sim \mathrm{B}^{2} \mathrm{v}_{\mathrm{a}}$, and so with $\mathrm{B}^{2} \sim \rho \ell_{\mathrm{P}}^{2} \Omega^{2}$ 
the associated power is:

$$
\mathrm{L}=\mathrm{r}^{2} \mathrm{~B}^{2} \mathrm{v}_{\mathrm{a}} \sim \mathrm{B}^{2} \mathrm{r}^{2} \ell_{\mathrm{P}} \Omega \sim \rho \mathrm{r}^{5} \Omega^{3}\left(\frac{\ell_{\mathrm{P}}}{r}\right)^{3} .
$$

Note that the next-to-last expression on the RHS is essentially just the characteristic Blandford-Payne luminosity; however, in this case the field is not externally given, but provided by the dynamo process so that the final expression on the RHS is given entirely in terms of local, internal quantities. The implication is that this amount of power is available in an axial, helical field without twisting an externally anchored field. Again, while this analysis has superficial resemblance to other jet mechanisms, it involves rather different physics and is self-contained. Whether this truly provides a jet remains to be seen.

Note that this process of creating a large scale field with an MRI-driven dynamo with its promise of naturally driving axial, helical flows does not require an equipartition field. As pointed out by Wheeler et al. (2002), the field does not have to have equipartition strength and hence to be directly dynamically important in order to be critical to the process of core collapse. The field only has to be significantly strong to catalyze the conversion of the free energy of differential rotation of the neutron star into jet energy. As long as this catalytic function is operative, the rotational energy should be pumped into axial flow energy until there is no more differential rotation. For the case of stellar collapse, this would seem to imply that, given enough rotational energy in the neutron star, this machine will work until there is a successful explosion. Even if the core collapses directly into a black hole, or does so after some fall-back delay, the basic physics outlined here, including magnetic helicity currents and their associated power should also pertain to black hole formation.

\section{Neutrino Transport}

Fields of order $10^{15}$ to $10^{16} \mathrm{G}$ that will characterize both neutron star and black hole formation may affect neutrino transport. With a large magnetic field, direct $\nu-\gamma$ interaction is possible mediated by $\mathrm{W}$ and $\mathrm{Z}$ bosons. This would allow neutrino Cerenkov radiation, $\nu \rightarrow \nu+\gamma$, and would enhance plasmon decay, $\gamma \rightarrow \nu+\nu$ (Konar 1997).

In addition, processes like $\nu \rightarrow \nu+\mathrm{e}^{+}+\mathrm{e}^{-}$would no longer be kinematically forbidden. In that case, closed magnetic flux loops can trap pairs. The energy in pairs would grow exponentially to the point where annhilation cooling would balance pair creation. Thompson \& Duncan (1993) 
estimated that an energy as much as $\mathrm{E}_{\text {pair }} \sim 10^{50}$ erg could be trapped in this way. This is not enough energy to cause a robust explosion, but it is enough energy to drive the dynamics of core collapse in a substantially different way, perhaps by inducing anisotropic flow if the flux loops are themselves distributed anisotropically.

With substantial magnetic fields, the cross section for inverse beta decay, $\nu_{\mathrm{e}}+\mathrm{n} \rightarrow \mathrm{p}+\mathrm{e}^{-}$, would become dependent on neutrino momentum, especially for asymmetric field distributions, which would be the norm (Lai \& Qian 1998; Bhattacharya \& Pal 2003; Ando 2003; Duan \& Qian 2004).

All these processes and more should be considered quantitatively in core collapse to form neutron stars and black holes.

\section{Recent Work on Magnetic Core Collapse}

In this section we will review, compare and contrast some recent work on rotating magnetic collapse and related issues that pertain to understanding asymmetric core collapse. Other relevant work that we do not discuss in detail is in Burrows \& Hayes (1996) and Lai et al. (2001) and references therein that discuss the effects of neutrino flux asymmetry. A crucial piece of information that can inform the discussion of potential MHD effects even in the absence of the explicit inclusion of magnetic fields is to give sufficient information from a rotating collapse to at least crudely estimate the saturation field according to the prescription $v_{a} \sim r \Omega$ or $B \sim \sqrt{4 \pi \rho} r \Omega$, that is, the angular velocity and the density profiles, or, even better, the product $\sqrt{\rho} \Omega$. Many studies of rotating collapse produce such information, but fail to present it explicitly. It would be very valuable if such information were presented explicitly as a function of time.

Akiyama et al. (2003) - As outlined above, Akiyama et al. did "shellular" rotating collapse calculations with multi-group flux limited diffusion of neutrinos, no angular momentum transport (although that possibility was discussed) and a heuristic treatment of the MRI. They concluded that core collapse is generically susceptible to the MRI and that the MRI could be important. They found fields of order $10^{15}$ to $10^{16} \mathrm{G}$ could plausibly be generated in tens of milliseconds after bounce. Such fields are interestingly larger than the QED limit, but still not directly dynamically important. The magnetic field need not necessarily be dynamically important if the field can catalyze the dumping of the rotational energy of the neutron star into some useful, explosion-inducing form, jets or otherwise. This basic energy requirement puts a premium on rapid rotation of the progenitor and 
the proto-neutron star in order to have a sufficiently large energy reservoir on which to draw. Of course, the rotational energy may be abetted by the large neutrino flux.

Thompson, Quataert, \& Burrows (2004) - Thompson et al. also did "shellular" rotating collapse calculations with a heuristic treatment of the MRI. Not surprisingly, given the similar assumptions and computations, they confirmed the field strength estimates of Akiyama et al. The new ingredient in this paper was to add viscous dissipation heating. Thompson et al. found that they could induce explosions for rapid enough rotation.

Fryer \& Warren (2004) - In a series of works culminating, for now, in this paper, Fryer \& Heger (2000) and Fryer \& Warren (2002) have explored rotating core collapse. See also Fryer's contribution to these proceedings. This work has used an SPH code with Fryer \& Heger and Fryer \& Warren examining the 2D case and Fryer \& Warren (2004) full 3D hydrodynamics. A feature that complicates the comparison of the results of this work with that from grid-based codes is that the SPH code yields prompt explosions in the basic non-rotating case, but no current grid-based code does so. The SPH code uses single energy, flux-limited diffusion. In the rotating calculations there are issues of angular momentum transport in SPH versus grid-based calculations. Fryer \& Heger (2000) and Fryer \& Warren (2002) found that rotation alone could induce bi-polar, asymmetric explosions with axis ratios of 2 to 1 , but the calculations were not run into the free-expansion phase, so it is not clear that this large asymmetry will survive as required by the spectropolarimetry.

In their 3D calculation, Fryer \& Warren take note of significant evolution in the angular momentum distribution. An important factor is the tendency for low angular momentum matter to flow in along the rotation axis while larger angular momentum matter tends to halt along the equator. This aspect of the dynamics cannot be captured in "shellular" calculations, but should be manifested in 2D calculations. Unfortunately, other papers have not commented specifically on this phenomenology which should be quite generic. It would be useful in making comparisons if others were to do so. Fryer \& Warren (2004) do not include MHD, but use the heuristic prescription of the saturation field strength presented by Akiyama et al. to estimate the field strength for the angular velocity gradient and density they compute. Their estimates of the field strength are substantially less than found by Akiyama et al. even though, despite the very different dynamics (3D versus "shellular"), the resulting angular velocity gradients are rather similar. The difference seems to be that, with a prompt explosion, the 
density declines rapidly, thus decreasing the implied Alfvén speed and hence saturation field for a given angular velocity gradient.

Buras, Rampp, Janka, \& Kifonidis (2003) - Buras et al. do a sophisticated rotating collapse with Boltzmann neutrino transport on radial rays. These calculations have no magnetic effects, but are of the sort that can establish the ambience in which MHD effects will occur. These calculations give bi-polar flow, but no explosion. The angular velocity profiles are not presented, so it is difficult to compare to other computations in that fundamental regard.

Ott, Burrows, Livne \& Walder (2003) - Ott et al. used Livne's VULCAN/2D code to study rotating collapse. They include no neutrino transport, but do present useful information on angular velocity profiles at certain epochs. These calculations revealed the strong shear expected in core collapse and gave bi-polar flow patterns, but no explosion.

Kotake, Sawai, Yamada \& Sato (2004) - Kotake et al. present 2D rotating, MHD collapse calculations using the ZEUS-2D code. They incorporate an approximate neutrino cooling with a leakage scheme. They assume the initial field prior to collapse is predominantly toroidal and explore the effect on anisotropic neutrino radiation. They find more effective neutrino heating near the axis in a way that affects the dynamics. These calculations assume rapid pre-collapse rotation and pre-collapse magnetic fields in the range $5 \times 10^{9}-10^{14} \mathrm{G}$. Such initial fields are probably unrealistically large. The calculations do produce phenomena that resemble MHD jets. The effects of field line wrapping are difficult to discriminate from the MRI, but Kotake et al. conclude that the MRI is likely to occur after bounce due to non-axisymmetric perturbations.

Yamada \& Sawai (2004) - Yamada \& Sawai also use ZEUS-2D but with a parametrized equation of state and no neutrinos. They assume rapid pre-collapse rotation and pre-collapse poloidal magnetic fields that are uniform, parallel to the rotation axis and with an amplitude of $\sim 10^{12}$ G. Again these large initial fields are probably unphysical. Yamada \& Sawai find large fields "behind the shock" not in the core as for the pioneering calculation of LeBlanc \& Wilson (1971). Once again it is difficult to see whether the growth of field strength is due to field line wrapping, especially with the initial axial field, or some aspect of the MRI, or both.

Madokoro, Shimizu, \& Motizuki (2003) - Madokoro et al. (see also Shimizu et al. 1994) explore non-rotating models in which a prolate, anisotropic neutrino radiation field is imposed. They find that such an anisotropic neutrino flux gives a larger explosion energy for given neutrino 
luminosity.

Ardeljan, Bisnovatyi-Kogan, Kosmachevskii \& Moiseenko (2004) - Ardeljan et al. (see also Ardeljan, Bisnovatyi-Kogan \& Moiseenko 2004 and Moiseenko, Bisnovatyi-Kogan \& Ardeljan 2004) present their own version of 2D MHD collapse and explosion for a collapsing bare white dwarf. They compute the collapse with rotation until the structure is nearly in hydrostatic equilibrium and then "turn on" a field that is subsequently amplified. They explore both dipole and quadrupole initial fields. The magnetic field subsequently grows to become comparable to the local pressure at which time an MHD shock is generated. The formation of the MHD shock may be related to the low density associated with the bare white dwarf collapse. They get some mass ejection with an energy of about $5 \times 10^{50}$ ergs for a model in which the initial magnetic energy is a fraction $10^{-6}$ of the gravitational energy. If the gravitational energy corresponds to a neutron star with binding energy of order $10^{53}$ ergs this corresponds to an initial field of roughly $10^{15} \mathrm{G}$. Although the MRI is mentioned in Ardeljan, Bisnovatyi-Kogan \& Moiseenko and by Moiseenko et al., few details are presented, so the mechanism of the field amplification is not clear. Unique among the calculations summarized here, this work follows the neutron star for $\sim 10 \mathrm{~s}$ as it contracts and speeds up.

Hawley \& Balbus (2002) - Hawley \& Balbus performed the first MHD simulation of a collapse-related environment in which the MRI and jet formation were explicit ingredients. They use the ZEUS algorithms to solve the MHD equations. This was a 3D MHD simulation of the accretion of a torus of matter around a black hole. This is not the same as a true collapse calculation in the sense that there is no surrounding star, but it is still instructive. The torus accretes due to the turbulent stresses generated by the MRI. The resulting flow forms a hot, thick, nearly-Keplerian disk, a surrounding magnetized corona, and a jet up the axis. A key point is that their jet is not confined by hoop stress. It is held out by the centrifugal barrier and held in by the pressure of the highly magnetic $(\beta<<1)$ corona. It is not clear how much this simulation would change if there were a surrounding, infalling star. Hawley \& Balbus note that there was no significant dynamical difference between simulations that included or omitted resistive heating. Hawley \& Balbus suggest that they get larger fields than in their closed box simulations, but do not discuss the reasons in any detail. They also do not explicitly discuss whether their fields are turbulent, changing sign on turbulent time scales, or are well-ordered and large scale.

Proga, MacFadyen, Armitage, \& Begelman (2003) - Proga et 
al. also explored a somewhat different problem, the rotating magnetic core collapse onto a black hole rather than onto a proto-neutron star, but the similarities and differences are again instructive. Proga et al. also employ ZEUS-2D MHD to do calculations of accretion of a helium envelope around a pre-existing black hole. They adopt a particular initial angular momentum distribution that does not necessarily correspond to the other collapse models discussed here. They adopt an initial field that is purely radial, again a configuration unlike any of the other work mentioned here. They find that a thick, Keplerian torus forms with the subsequent development of a Poynting-flux dominated jet. They argue that the MRI is active in producing amplification of their initial field, but give few details of the operation of the MRI in their models. It is of particular interest that Proga et al. find their jet to be Poynting-flux dominated, since none of the canonical neutron star formation calculations seem to do so, even with rather large initial rotation and magnetic fields. This may be an artifact of their doing a calculation with a previously existing black hole onto which the helium envelope is dumped "impulsively." This may lead to a more evacuated, low density environment within the collapsing core and hence perhaps a tendency to be more nearly field dominated at a given field strength than if the collapse were followed $a b$ initio from an iron core. There may also be some differences in the way field amplification evolves in this environment. There could also be differences in the intrinsic environment of proto-neutron stars versus black holes. It would be very interesting to explore in more detail the differences between the calculation of Proga et al. and that of, for instance, Kotake et al. (2004).

This summary raises a central practical issue. The grand goal is to do a full 3D, sufficiently resolved (whatever that means) simulation of rotational magnetic core collapse with anisotropic neutrino transport. Various groups, as just summarized, are taking the first steps in this direction. To what degree can current (or future) simulations be regarded as adequate to capture the physics? A case in point are simulations that may or may not reproduce the physics of the MRI.

A basic issue here is well-illustrated by Balbus \& Hawley (1998) who point out that the Cowling dynamo theorem pertains to 2D MHD calculations. This theorem states that an axially-symmetric magnetic field cannot be produced by fluid flow (Cowling 1957). The implication, as pointed out by Balbus \& Hawley (1998), is that sustained magnetic field amplification by axisymmetric turbulence is impossible in an isolated dissipative 2D system. Balbus \& Hawley show, as pointed out earlier, that the behavior of 
MRI simulations with initial vertical field and those with initial turbulent field $(<\mathrm{B}>=0)$ are very different with the former yielding channel flow solutions and the latter turbulent solutions. In $2 \mathrm{D}$ simulations, however, both configurations yield a decaying field after an initial transient growth phase. Three-dimensional simulations, on the other hand, show a rapid growth to a saturation field. Balbus \& Hawley argue that the results still retain some memory of the initial field configuration, but that the MRI works to grow the field exponentially rapidly even with $\langle\mathrm{B}>=0$ as long as the simulation is adequately resolved. The time to reach saturation depends on the initial value of $\left\langle\mathrm{B}^{2}>^{1 / 2}\right.$, but the saturation level is basically independent of the value of the initial mean field.

What, then, are we to make of the various simulations described above, especially those that are in 2D and may or may not see the effects of the MRI? It is possible that they are seeing the transient growth before the Cowling anti-dynamo effects set in. Is that adequate for a dynamical situation like a supernova where the field only needs to act long enough to produce an explosion? Perhaps, but that does not seem satisfactory when the goal is to get the physics right. In addition, the $2 \mathrm{D}$ simulations reproduced in Balbus \& Hawley (1998) seem to show smaller ratios of peak fields to initial fields than the $3 \mathrm{D}$ calculation and that the field in $2 \mathrm{D}$ simulations decays on a timescale comparable to the rotation time, so there are indications already that one should not trust any $2 \mathrm{D}$ simulation to properly capture the physics of the MRI. We have also seen that various groups have made radically different assumptions about the initial field structure - axial, toroidal, radial - when the simulations indicate that the initial field configuration may affect the results, especially in 2D. There has been no systematic study of this possibility.

Another point to ponder is the stricture in the Cowling dynamo theorem that the system to which the theorem applies should be isolated and dissipative. A supernova is dissipative, but it is not isolated; the whole point is to eject matter through previously existing boundaries. The possibility of magnetic helicity currents as described by Vishniac \& Cho $(2001 ; \S 6)$ may also be relevant in this context. Is it possible that MHD calculations that produce jets and outflow are thereby not "isolated," and not subject to the anti-dynamo restrictions, and hence in some sense more trustworthy? Again this point has not been discussed at all in the literature.

This is not to deny that all the calculations described above have merit as this difficult regime is explored. The lesson is clear, however that the $\mathrm{MRI}$ is tricky to simulate and care must be taken in computing, presenting, 
and comparing results among various simulations.

\section{Conclusions}

Spectropolarimetric studies have shown that all core-collapse supernovae yet observed are significantly asymmetric, with geometries that can be predominantly bi-polar, but can also be more complex in ways that have yet to be sufficiently characterized or understood (see Wang et al. 2001, Wang et al. 2003b). This data, along with direct imaging of objects like SN 1987A, the Crab nebula and Cas A, means that the dynamics and the radiative transfer, both photons and neutrinos, are very likely to be significantly asymmetric. Account of this asymmetry must be made in the analysis of these events, including the derivation of such basic quantities as the ejecta mass and energy.

Core collapse is an intrinsically shearing environment and hence generically subject to dynamo-like instabilities such as the magneto-rotational instability. This means that both rotation and magnetic fields are intrinsic to the process of core collapse. This applies both to supernovae and to gamma-ray bursts, to the formation of neutron stars and of black holes.

Many of the points of this discussion have been made with the implicit assumption that the cause of the asymmetry of core collapse supernovae as revealed by the spectropolarimetry is in some way related to rotation which must be accompanied by some dynamo action and field growth. A last point concerns alternatives to this tacit assumption. Blondin, Mezzacappa, \& DeMarino (2003) have shown that standing spherical accretion shocks such as those that arise in non-rotating, non-magnetic core-collapse supernova models are unstable to $\ell=1$ and 2 modes of oscillation. See also Blondin in these proceedings, Foglizzo (2002), and the calculations of full collapse with neutrino transport illustrating this instability described by Janka (2004) and by Scheck et al. (2004). This instability and the rapid growth of turbulence behind the shock is driven by the injection of vorticity as the shock is perturbed from spherical. This interesting result means that one must, at least, be cautious about interpreting bi-polar symmetry as the result of rotation. On the other hand, sufficient rotation (never mind magnetic fields) may damp this particular instability (but see Scheck et al.). It is perhaps worth noting that this instability can only produce bi-polar structure whereas the observations show that strong deviations from this simple geometry are not uncommon. Of course, the simplest jet-induced models are also intrinsically axially symmetric and fail the same test. Clearly, this 
instability must be added to the zoo of possible phenomenology and treated self-consistently in future work.

The bottom line is that many new vistas have been opened by considering the intrinsic asymmetry of core-collapse supernovae and the ability of rotating, magnetic collapse to account for the observations. There is much work to be done to understand all the attendant physics.

\section{Acknowledgments}

The authors are grateful to Tony Mezzacappa for his invitation and encouragement to attend this workshop, to the Institute for Nuclear Theory for providing a rich and fruitful environment, and to John Hawley and Chris Fryer for especially rewarding conversations at the meeting. They also thank Dave Meier and Peter Williams for valuable on-going discussion of these topics. JCW is deeply grateful for the contributions of his collaborators Lifan Wang, Peter Höflich and Dietrich Baade and the excellent staff at the VLT, all of whom were critical to the success of our spectropolarimetry program. This work was supported in part by NASA Grants NAG5-10766 and NAG5-9302 and by NSF Grant AST-0098644.

\section{References}

1. Ando, S. 2003, Phy. Rev. D, 68, 63002

2. Akiyama, S. Wheeler, J. C., Meier, D. \& Lichtenstadt, I, ApJ, 584, 954

3. Akiyama, S., Wheeler, J. C., Meier, D. L., \& Duncan, R. C. 2004, in Cosmic Explosions in Three Dimensions, eds. P. Höflich, P. Kumar, \& J. C. Wheeler (Cambridge: Cambridge University Press), in press

4. Ardeljan, N. V. Bisnovatyi-Kogan, G. S., \& Moiseenko, S. G. 2004, MNRAS, in press (astro-ph/0410234)

5. Ardeljan, N. V. Bisnovatyi-Kogan, G. S., Kosmachevskii, K. V. \& Moiseenko, S. G. 2004, Astronomy, 47, 37.

6. Balbus, S. A. \& Hawley, J. F. 1991, ApJ 376, 214

7. Balbus, S. A. \& Hawley, J. F. 1998, Review of Modern Physics, 70, 1

8. Bhattacharya, K. \& Pal, P. B. 2003, hep-ph/0209053

9. Blackman E. G. \& Brandenburg, A. 2002, ApJ, 579, 359

10. Blackman, E. G. \& Field, G. B. 2000, ApJ, 534, 984

11. Blackman, E. G. \& Field, G. B. 2002, Phys. Rev. Lett., 89, 265007

12. Blackman E. G. \& Tan, J. 2003, in "Proceedings of the International Workshop on Magnetic Fields and Star Formation: Theory vs. Observation," in press (astro-ph/0306424)

13. Blandford, R. D. \& Payne, D. G. 1982, MNRAS, 199, 833

14. Blondin, J. M., Mezzacappa, A., \& DeMarino, C. 2003, ApJ, 584, 971

15. Brandenburg, A., Blackman E. G. \& Sarson, G. R. 2003, Adv. Space Sci., 32,1835 
16. Buras, R., Rampp, M., Janka, H.-T., \& Kifonidis, K. 2003, Phys. Rev. Lett., 90, 241101

17. Burrows, A., \& Hayes, J., 1996, Phys. Rev. Lett., 76, 352

18. Cowling, T. G. 1957, Magnetohydrodynamics, (New York: Interscience)

19. Duan, H., \& Qian, Y. -Z. 2004, Phys. Rev. D, 69, 123004

20. Duan, H. 2004, private communication

21. Duncan, R. C. \& Thompson, C. 1992, ApJ, 392, L9

22. Fesen, R. A. 2001, ApJ Supp. , 133, 161

23. Field, G. B. \& Blackman E. G. 2002, ApJ, 572, 68

24. Foglizzo, T. 2002, A\&A, 392, 353

25. Fryer, C. L. \& Heger, A. 2000, ApJ, 541, 1033

26. Fryer, C. L. \& Warren, M. S. 2002, ApJ Lett., 574, L65

27. Fryer, C. L. \& Warren, M. S. 2004, ApJ, 601, 391

28. Gruzinov, A. V. \& Diamond, P. H. 1994, Phys. Rev. Lett., 72, 1651

29. Hawley, J. F. \& Balbus, S. A. 2002, ApJ, 573, 738

30. Hjorth, J. et al. 2003, Nature, 423, 847

31. Höflich 1991, Astron \& Astrophys.,, 246, 481;

32. Höflich, P., Khokhlov, A. \& Wang, L. 2001, in Proc. of the 20th Texas Symposium on Relativistic Astrophysics, eds. J. C. Wheeler \& H. Martel, (New York: AIP), 459

33. Howell, D. A., Höflich, P., Wang, L., \& Wheeler, J. C. 2001, ApJ, 556, 302

34. Hwang, U. et al. 2004, ApJ Lett., 615, L117

35. Kawabata, et al. 2003, ApJ, 593, L19

36. Khokhlov, A. \& Höflich, P. 2001, in Explosive Phenomena in Astrophysical Compact Objects, eds. H.-Y, Chang, C.-H. Lee \& M. Rho, AIP Conf. Proc. No. 556, (New York: AIP), p. 301

37. Khokhlov A.M., Höflich P. A., Oran E. S., Wheeler J.C. Wang, L, \& Chtchelkanova, A. Yu. 1999, ApJ, 524, L107

38. Kleeorin, N. I., Moss, D., Rogachevskii, I. \& Sokoloff, D. 2002, A\&A, 387, 453

39. Konar, S. 1997, PhD. Thesis

40. Kotake, K., Yamada, S., \& Sato, K. 2003, ApJ, 595, 304

41. Kotake, K., Sawai, H., Yamada, S., \& Sato, K. 2004, ApJ, 608, 391

42. Kulsrud, R. M. \& Anderson, S. W. 1992, ApJ, 396, 606

43. Lai, D. \& Qian, Y.-Z. 1998, ApJ, 505, 844

44. Lai, D., Chernoff, D. F. \& Cordes, J. M. 2001, ApJ, 549, 1111

45. Leonard, D. C., Filippenko, A. V., Barth, A. J., \& Matheson, T. 2000, ApJ, 536,239

46. Leonard, D. C. \& Filippenko, A. V. 2001, PASP, 113, 920

47. Leonard, D. C., Filippenko, A. V., Ardila, D. R., \& Brotherton, M. S. 2001, ApJ, 553, 861

48. Leonard, D. C., Filippenko, A. V., Chornock, R. \& Foley, R. J. 2002, PASP, 114,1333

49. MacFadyen, A. \& Woosley, S. E. 1999, ApJ, 524, 262

50. Maeda, K., Nakamura, T., Nomoto, K., Mazzali, P., Patat, F. \& Hachisu, I. 2002, ApJ, 565, 405 
51. Madokoro, H., Shimizu, T., \& Motizuki, Y. 2003, ApJ, 592, 1035

52. Marietta, E., Burrows, A., \& Fryxell, B. 2000, ApJS, 128, 615

53. Meier, D. L., Koide, S. \& Uchida, Y. 2001, Science, 291, 84

54. Moiseenko, S. G., Bisnovatyi-Kogan, G. S. \& Ardeljan, N. V. 2004, in "16042004 Supernovae as Cosmological Lighthouses," eds. M. Turatto et al. (San Francisco: ASP) astro-ph/0410330

55. Nagataki, S., Mizuta, A., Yamada, H., Takabe, H. \& Sato, K. 2003, ApJ, ApJ, 596, 401

56. Ott, C. D., Burrows, A., Livne, E., \& Walder, R. 2004, ApJ, 600, 834

57. Proga, D., MacFadyen, A. I., Armitage, P. J., \& Begelman, M. C. 2003, ApJ Lett., 599, L5

58. Scheck, L., Plewa, T., Janka, H. Th., Kifonidis, K. \& Müller, E. 2004, Phys, Rev. Lett., 92, 011103-1

59. Shimizu, T., Yamada, S., \& Sato, K. 1994, ApJ. Lett., 432, L119

60. Stanek, K. Z. et al. 2003, ApJ, 591, L17

61. Thompson, C. \& Duncan, R. C. 1993, ApJ, 408, 194

62. Thompson, T. A., Quataert, E., \& Burrows, A. 2004, preprint (astroph/0403224)

63. Thorstensen, J. R., Fesen, R. A. \& van den Bergh, S. 2001, ApJ, 122, 297

64. Vishniac, E. T. \& Cho, J. 2001, ApJ, 550, 752

65. Wang, L., Baade, D., Höflich, P. \& Wheeler, J. C. 2002b, ESO Messenger, No. 109, 47

66. Wang, L., Howell, D. A., Höflich, P., \& Wheeler, J. C. 2001, ApJ, 550, 1030

67. Wang, L., Wheeler, J. C., \& Höflich, P. 1997, ApJ. Lett., 476, L27

68. Wang, L., Wheeler, J. C., Li, Z., \& Clocchiatti, A. 1996, ApJ, 467, 435

69. Wang, L. et al. 2002a, ApJ, 579, 671

70. Wang, L. et al. 2003a, ApJ, 591, 1110

71. Wang, L. et al. 2003b, ApJ, 592, 457

72. Wang, L., Baade, D., Höflich, P., Wheeler, J. C., Kawabata, K., \& Nomoto, K. 2004, ApJ Lett., 604, L53

73. Wheeler, J. C. 2004, in Cosmic Explosions in Three Dimensions: Asymmetries in Supernovae and Gamma-Ray Bursts, eds P. Höflich, P. Kumar \& J. C. Wheeler (Cambridge: Cambridge University Press), astro-ph/0401323

74. Wheeler, J. C., Meier, D. L. \& Wilson, J. R. 2002, ApJ, 568, 807

75. Wheeler, J. C., Yi, I., Höflich, P. \& Wang, L. 2000, ApJ, 537, 810

76. Williams, P. T., 2003, IAOC Workshop "Galactic Star Formation Across the Stellar Mass Spectrum," ASP Conference Series," ed. J. M. De Buizer, in press (astro-ph/0206230)

77. Yamada, S. \& Sawai, H. 2004, ApJ, 608, 907 